\title{
NOTE \\ Effect of High-fiber Diet on Plasma High Density Lipoprotein (HDL) Cholesterol Level in Streptozotocin-induced Diabetic Rats
}

\author{
Sono YAMASHITA and Kamejiro YAMASHITA \\ Department of Medicine, University of Tsukuba, \\ Niiharigun, Ibaraki-ken, 305, Japan
}

\begin{abstract}
We have recently reported that high-fiber diet is an effective therapeutic regimen for the control of some metabolic derangements in diabetic rats (Yamashita et al., 1980). Additional effects of high-fiber diet on the metabolic states in streptozotocininduced diabetic rats were investigated. The animals were divided into two groups : a dietary fiber group fed on diet containing $10 \%$ dietary fiber ad libitum and a control group fed on a control diet ad libitum for fifty days. Fasting blood glucose levels were significantly lower in rats in the dietary fiber group than in those in the control group. In addition, plasma high density lipoprotein (HDL) cholesterol values and the HDL cholesterol/total cholesterol ratio in fiber-fed rats were higher than those in the control group. These results indicate that a high-fiber diet may have a beneficial effect on the metabolism of cholesterol as well as glucose in diabetics.
\end{abstract}

Trowell (1972) and Burkitt, Walker and. Painter (1974) suggested that a fiber-depleted diet may play a causative role in the development of clinical diabetes millitus. Thereafter, several lines of clinical observation indicated that a high-fiber diet was useful means of alleviating the metabolic derangement in diabetics (Jenking et al., 1975; Miranda \& Horwitz, 1978). Recently, Chen et al. (1979) reported that rats fed sucrose-cholesterol-guar gum diet had lower plasma total cholesterol, liver triglyceride and liver cholesterol, but higher plasma high density lipoprotein (HDL) cholesterol values than those fed a sucrose-cholesterol diet. We have also reported that diabetic rats fed a high-fiber (bagasse) diet had lower blood glucose and plasma triglyceride levels than those fed a control diet (Yama-

\section{Received May 10, 1980.}

Address repint requests to: Kamejiro Yamashita, M. D. Department of Medicine, University of Tsukuba. Niiharigun, Ibaraki-ken, 305 Japan. shita et al., 1980). In this study, evidence is presented that plasma high density lipoprotein (HDL) cholesterol values were increased in rats fed high-fiber diet.

\section{Materials and Methods}

Male Wistar-strain rats weighing $180-200 \mathrm{~g}$ were housed in individual cages. Diabetes was induced by a single injection of streptozotocin $(60 \mathrm{mg} / \mathrm{kg}$ of body weight) into the tail vein. Streptozotocin was dissolved Immediately before use in a citric acid buffer $(50 \mathrm{~mm})$ adjusted to $\mathrm{pH} 4.5$ and $0.3 \mathrm{ml}$ of the solution was injected. After the diabetic rats were fed with the control diet for one week, only those rats with fasting plasma glucose of $180 \mathrm{mg} / \mathrm{d} l$ or higher were used in the study. They were divided into two groups (ten rats in each group). Rats in the dietary fiber group were fed on a diet containing $10 \%$ dietary fiber ad libitum. Rats in the control group were fed on a control diet ad libitum for fifty days. The diet was given between 3 and 5 p.m. every day. The composition of the control diet was $66.85 \% \alpha$-cornstarch, $5 \%$ glucose, $18 \%$ casein, $5 \%$ corn oil, $4 \%$ minerals, $1 \%$ vitamins and $0.15 \%$ choline chloride. The composition of the diet containing 
$10 \%$ dietary fiber was $56.85 \% \quad \alpha$-cornstarch, $10 \%$ dietary fiber (Solka-Floc, BW-200: manufactured from cellulose, Brown Co. U.S.A.) and the other elements mentioned above.

After fasting for $10 \mathrm{hr}$, the rats were anesthetized with pentobarbital $(50 \mathrm{mg} / \mathrm{kg}$, ip) and $1 \mathrm{ml}$ of blood was withdrawn from the jugular vein. Blood glucose was determined by a glucose oxidase method. Plasma high density lipoprotein (HDL) cholesterol was assayed using a kit provided by Dai-ichi Chemical Co., and total cholesterol was determined by the method of Zak-Henly (Zak, 1957). Salka-Floc, BW200 was kindly provided by Mr. Sasayama, Pfizer Co.

\section{Results and Discussion}

Figure 1 demonstrates that the mean blood glucose level of rats on the high-fiber diet remained almost unchanged throughout the experimental periad, while the level of those on control diet was significantly higher than that observed in rats fed the high-fiber diet. These results are consistent with our previous report (Yamashita et al., 1980) in a similar experiment employing bagasse as the fiber, in which the mean blood glucose level even on the high-fiber diet was gradually increased. The somewhat different result between them is probably due to the quality of the fiber used in the experiments.

The cholesterol lowering effect of dietary fiber has been reported (Jenkins et al., 1975, Chen et al., 1979), but uniformly has not been achieved (Heaton \& Pomare, 1974, Raymond et al., 1977). In the present study, we obtained the results showing that plasma cholesterol levels on the highfiber diet (Group A) were not significantly different from those on the control diet (Group B). The plasma total cholesterol values in Group A on 0, 10, 20, 30, 40 and 50 days were $76 \pm 7,72 \pm 8,76 \pm 11,76 \pm$ $10,83 \pm 10,85 \pm 14$ and those in Group B were $82 \pm 8,76 \pm 10,77 \pm 8,84 \pm 9,81 \pm 8$, $82 \pm 9(\mathrm{mg} / \mathrm{d} l$, and the average \pm S.E. in each group, $\mathrm{n}=10)$. These results are different from those of Chen et al. (1979).

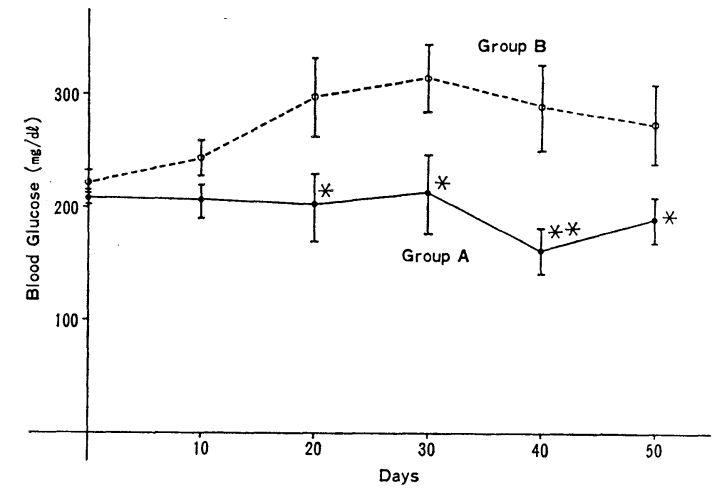

Fig. 1. Effect of high-fiber diet on fasting blood glucose level in streptozotocin-induced diabetic rats. After the diabetic rats were fed with the control diet for one week, they were divided into two groups (ten rats in each group). Rats in Group A were fed on a high-fiber diet ad libitum. Rats in Group B were fed on a control diet ad libitum. The composition of diets is mentioned in Materials and Methods. The period of the test is indicated on the abscissa. The values in the figure are expressed as the average \pm S.E. for each group $(n=10)$ and the values for Group $A$ on 20 , 30,40 and 50 days are significantly lower than those for their counterparts in Group B $\left(^{*} \mathrm{p}<0.05\right.$, ** $\mathrm{p}<0.01$ by Student's $t$-test.

They observed the lowering effect of highfiber diet on plasma total cholesterol levels. However, they employed cholesterol-rich diets to get high levels of plasma total cholesterol. Therefore the reason why the present results different from theirs may be due to either the difference in the experimental procedures or the quality of the fiber which we employed. Plasma HDL cholesterol levels in diabetic rats were lower than those in normal rats $(30 \pm 8 \mathrm{mg} / \mathrm{d} l)$. Figure 2 shows that plasma HDL cholesterol levels on the high-fiber diet were gradually increased, reaching the normal range after 40 days and they were significantly higher than those on the control diet (Fig. 2 upper panel). However, the exact mechanism in which the high-fiber diet causes an increase in the HDL cholesterol level has not yet been elucidated. 


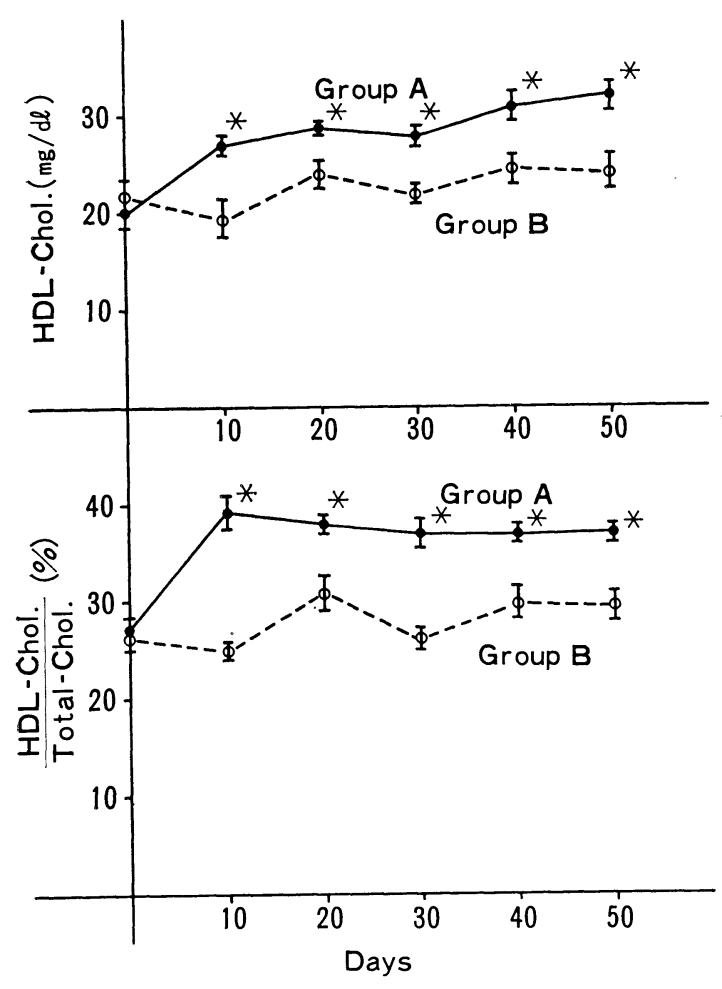

Fig. 2. Effect of high-fiber diet on plasma HDLcholesterol level (upper panel) and HDL-cholesterol/ total cholesterol ratio (lower panel) in streptozotocin-induced diabetic rats. The experimental procedures were as described under Methods and in the legend to Fig. 1. The values in the figures are expressed as the average $\pm S$. E. for each group $(n=10)$ and the values for the plasma HDLcholesterol level (upper panel) and HDL-cholesterol/ total cholesterol (lower panel) in Group $A\left({ }^{*}\right)$ on $10,20,30,40$, and 50 are significantly higher than those for their counterparts in Group $B(p<0.01)$.

Plasma HDL cholesterol/total cholesterol ratio in the rats fed high-fiber diets was significantly higher than that on the control diet (Fig. 2 lower panel). Chen et al. (1979) reported that sucrose-cholesterol diet containing guar gum increased plasma HDL cholesterol to result in a higher HDL cholesterol/total cholesterol ratio. The present results are in agreement with their observations in that plasma HDL cholesterol and HDL cholesterol/total cholesterol ratio were increased in the high-fiber diet group.

Several lines of observation demonstrated a negative correlation between plasma HDL cholesterol (or HDL cholesterol/total cholesterol ratio) and atherosclerotic changes (Castelli et al., 1977; Gordon et al., 1977; Chen et al., 1977). From this point of view, the present finding that high-fiber diets raise the concentration of plasma HDL cholesterol indicates that high-fiber diets have a beneficial effect when there is metabolic derangement in diabetics.

\section{References}

Burkitt, D. P., A. R. Walker and N. S. Painter (1974). J. Am. Med. Assoc. 229, 1068.

Castelli, W. P., J. T. Doyle, T. Gordon, C. G. Hames, M. C. Hjortland, S. B. Hulley, G. K. Kagan and W. J. Zukel (1977). Circulation 55, 767.

Chen, Wen-Ju Lin and J. W. Anderson (1979). J. Nutr. 109, 1028.

Gordon, T., W. P. Castelli, M. C. Hjortland, W. B. Kannel and T. R. Dawber (1977). Am. J. Med. 62, 707.

Heaton, K. W., and E. W. Pomare (1974). Lancet $1,49$.

Jenkins, D. J. A., T. M. S. Wolever, A. R. Leeds, M. A. Gassull, P. Haisman, J. Dilawari, D. V. Goff, G. L. Metz and K. G. M. M. Alberti (1978). Br. Med. J. 1, 1392.

Jenkins, D. J. A., A. R. Leeds, C. Newton and J. H. Cummings (1975). Lancet 1, 1116.

Miranda, P. M. and D. L. Horwitz (1978). Ann. Int. Med. 88, 482.

Raymond, T. L., W. E. Connor, D. S. Lin, S. Warner, M. M. Fry and S. Connor (1977). J. Clin. Invest. 60, 1429.

Trowell, H. (1972). Am. J. Clin. Nutr. 25, 926.

Yamashita, S. and K. Yamashita (1980). Endocrinol. Japon. 2, 169.

Zak, B. (1957). Am. J. Clin. Pathol. 27, 583. 